\title{
Local Unitary Equivalence of Multi-qubit Mixed quantum States
}

\author{
Ming $\mathrm{Li}^{1,2}$, Tinggui Zhang ${ }^{2}$, Shao-Ming $\mathrm{Fei}^{2,3}$, Xianqing Li-Jost ${ }^{2}$, and Naihuan Jing ${ }^{2,4,5}$ \\ ${ }^{1}$ School of Science, China University of Petroleum, 266580 Qingdao, China \\ ${ }^{2}$ Max-Planck-Institute for Mathematics in the Sciences, 04103 Leipzig, Germany \\ ${ }^{3}$ Department of Mathematics, Capital Normal University, 100037 Beijing, China \\ ${ }^{4}$ Department of Mathematics, North Carolina State University, Raleigh, NC27695, USA \\ ${ }^{5}$ School of Science, South China University of Technology, Guangzhou 510640, China
}

\begin{abstract}
We present computable criterion for completely classifying multi-qubit quantum states under local unitary operations. The criterion can be used to detect whether two quantum states in multi-qubit systems are local unitary equivalent or not. Once obtaining the positive answer, we are further able to compute the corresponding unitary operators precisely. Since the scheme is based on the mean values of some quantum mechanical observables, it supplies an experimental way to judge the local equivalence of quantum states.
\end{abstract}

PACS numbers: 03.67.-a, 02.20.Hj, 03.65.-w

Introduction: Quantum entangled states have become the most important physical resource in the rapidly developing field of quantum information science [1]. In particular, multipartite quantum entanglement plays key roles in many quantum information processing like oneway quantum computing, quantum error correction and quantum secret sharing [2 -5]. However, the nonlocal properties of multipartite mixed states are much more difficult to clarify than bipartite or pure states. Since two quantum states will be of the same power in implementing quantum information processing if they can be transferred to each other by local unitary (LU) transformations, and many crucial characters such as the degree of entanglement [6, 7], the maximal violations of Bell inequalities [8 11], and the teleportation fidelity [12, 13] remain invariant under the LU transformations, it has been an important problem to give a complete and operational classification of quantum states under LU transformations.

In principle, the LU equivalence problem can be characterized by the complete set of invariants under local unitary transformations. The author in [14] presented a complete set of 18 polynomial invariants for the locally unitary equivalence of two-qubit mixed states. Nice results have been obtained for three qubits states [15, 16], some generic mixed states [17 19], tripartite pure and mixed states [20]. In [21], Kraus presented a way to determine the local unitary equivalence of $n$-qubit pure states, which is extended to general multipartite pure states of arbitrary dimensions 22].

Mixed states are more general physically. For bipartite mixed quantum systems, Zhou et.al [23] have solved the local unitary equivalence problem by presenting a complete set of invariants such that two density matrices are locally equivalent if and only if all these invari- ants have equal values in these density matrices, which is operational for non-degenerate states. In [24], we have investigated the LU equivalence problem in terms of matrix realignment and partial transposition. A necessary and sufficient criterion for the local unitary equivalence of multipartite states, together with explicit forms of the local unitary operators have been presented. The criterion is shown to be operational for states having eigenvalues with multiplicity of no more than 2. However, as the two criteria above depend on the eigenvectors of density matrices, for degenerate states(especially for those with degeneracy larger than 2), they are less operational, similar to the multipartite pure state case when the reduced density matrices or the co-tensor matrices are degenerate.

In this paper, we investigate LU equivalence criterion and classification for general multipartite mixed quantum states. Alternatively, we deal with problem in terms of the generalized Bloch representations of density matrices. Hence the results work no matter the density matrices are degenerate or not. In particular, we present a both sufficient and necessary LU criterion for multi-qubit systems. To show that the criterion is operational, we give a detailed process in classifying multi-qubit mixed states under LU transformations. Moreover, our criterion can not only verify the LU equivalence of two mixed states, but also gives rise to the corresponding LU operators that transform one state to another LU equivalent one.

Local unitary equivalence for multipartite states: We first consider the local unitary equivalence problem for general $H_{1} \otimes H_{2} \otimes \cdots \otimes H_{N}$ quantum systems with $\operatorname{dim} H_{i}=d_{i}, i=1,2, \cdots, N$. A multipartite state $\rho \in H_{1} \otimes H_{2} \otimes \cdots \otimes H_{N}$ can be generally expressed in terms of the $S U(n)$ generators $\lambda_{\alpha_{k}}$ [26], 


$$
\rho=\frac{1}{\Pi_{i}^{N} d_{i}} \otimes_{j}^{N} I_{d_{j}}+\sum_{M=1}^{N} \sum_{\left\{\mu_{1} \mu_{2} \cdots \mu_{M}\right\}} \sum_{\alpha_{1} \alpha_{2} \cdots \alpha_{M}} \mathcal{T}_{\alpha_{1} \alpha_{2} \cdots \alpha_{M}}^{\left\{\mu_{1} \mu_{2} \cdots \mu_{M}\right\}} \lambda_{\alpha_{1}}^{\left\{\mu_{1}\right\}} \lambda_{\alpha_{2}}^{\left\{\mu_{2}\right\}} \cdots \lambda_{\alpha_{M}}^{\left\{\mu_{M}\right\}}
$$

where $I_{d_{j}}$ denotes $d_{j} \times d_{j}$ identity matrices, $\lambda_{\alpha_{k}}^{\left\{\mu_{k}\right\}}=I_{d_{1}} \otimes$ $I_{d_{2}} \otimes \cdots \otimes I_{d_{\mu_{k}-1}} \otimes \lambda_{\alpha_{k}} \otimes I_{d_{\mu_{k}+1}} \otimes \cdots \otimes I_{d_{N}}$, with $\lambda_{\alpha_{k}}$ appearing at the $\mu_{k}$ th position, and

$$
\mathcal{T}_{\alpha_{1} \alpha_{2} \cdots \alpha_{M}}^{\left\{\mu_{1} \mu_{2} \cdots \mu_{M}\right\}}=\frac{\prod_{i=1}^{M} d_{\mu_{i}}}{2^{M}} \operatorname{Tr}\left[\rho \lambda_{\alpha_{1}}^{\left\{\mu_{1}\right\}} \lambda_{\alpha_{2}}^{\left\{\mu_{2}\right\}} \cdots \lambda_{\alpha_{M}}^{\left\{\mu_{M}\right\}}\right],
$$

which can be viewed as the entries of the tensors $\mathcal{T}\left\{\mu_{1} \mu_{2} \cdots \mu_{M}\right\}$.

Before showing how to detect and classify quantum states under local unitary equivalence, we give a short review of high order singular value decomposition developed in [27]. For any tensor $\mathcal{T}$ with order $d_{1} \times d_{2} \times \cdots \times$ $d_{N}$, there exists a core tensor $\Sigma$ such that

$$
\mathcal{T}=U^{(1)} \otimes U^{(2)} \otimes \cdots \otimes U^{(N)} \Sigma,
$$

where $\Sigma$ forms a same order tensor with $\mathcal{T}$. To calculate the core tensor $\Sigma$, one first expresses $\mathcal{T}$ in matrix unfolding form $\mathcal{T}_{n}$. Then one derives the singular value decomposition of the matrix $\mathcal{T}_{n}=U^{(n)} \Lambda^{(n)} V^{(n)}$. The core tensor is then constructed by

$$
\Sigma=\otimes_{n=1}^{N} U^{(n) \dagger} \mathcal{T} .
$$

Let $\rho$ and $\rho^{\prime}$ be two mixed states in $H_{1} \otimes H_{2} \otimes \cdots \otimes H_{N}$. They are local unitary equivalent if

$$
\rho^{\prime}=\left(U_{1} \otimes \ldots \otimes U_{N}\right) \rho\left(U_{1} \otimes \ldots \otimes U_{N}\right)^{\dagger}
$$

for some unitary operators $U_{i}, i=1,2, \ldots, N$, where $\dagger$ denotes transpose and conjugate.
Proposition: If $\rho$ and $\rho^{\prime}$ are local unitary equivalent, then their corresponding tensors $\mathcal{T}\left\{\mu_{1} \mu_{2} \cdots \mu_{M}\right\}$ and $\left(\mathcal{T}^{\prime}\right)^{\left\{\mu_{1} \mu_{2} \cdots \mu_{M}\right\}}$ must have the same core tensor up to the local symmetry $\otimes_{n=1}^{M} P_{\left\{\mu_{1} \mu_{2} \cdots \mu_{M}\right\}}^{\mu_{n}}$ for any $\left\{\mu_{1} \mu_{2} \cdots \mu_{M}\right\} \subset\{1,2, \cdots, N\}$, where $P_{\left\{\mu_{1} \mu_{2} \cdots \mu_{M}\right\}}^{\mu_{n}}$ are block-diagonal matrices with each block being orthogonal matrix of size equal to the degeneracies of the singular values corresponding to the $\mu_{n}$ order unfolding of the tensor $\mathcal{T}^{\left\{\mu_{1} \mu_{2} \cdots \mu_{M}\right\}}$.

Proof: Assume $\rho$ and $\rho^{\prime}$ are related by (44). Note that for any given unitary operator $U, U \lambda_{i} U^{\dagger}$ is a traceless Hermitian operator which can be expanded according to the $S U(d)$ generators,

$$
U \lambda_{i} U^{\dagger}=\sum_{j=1}^{d^{2}-1} \frac{1}{2} \operatorname{Tr}\left\{U \lambda_{i} U^{\dagger} \lambda_{j}\right\} \lambda_{j} \equiv \sum_{j=1}^{d^{2}-1} O_{i j} \lambda_{j} .
$$

The entries $O_{i j}$ defines a real $\left(d^{2}-1\right) \times\left(d^{2}-1\right)$ matrix $O$. From the completeness relation of $S U(d)$ generators

$$
\sum_{j=1}^{d^{2}-1}\left(\lambda_{j}\right)_{k i}\left(\lambda_{j}\right)_{m n}=2 \delta_{i m} \delta_{k n}-\frac{2}{d} \delta_{k i} \delta_{m n}
$$

one can show that $O$ is an orthogonal matrix with determinant +1 , i.e. $O$ belongs to special orthogonal matrix group $S O(d)$. According to (1) and (5), we get that there must exist orthogonal operators $O^{1}, O^{2}, \cdots, O^{N}$ such that the following equations hold,

$$
\begin{aligned}
\rho^{\prime} & =\frac{1}{\Pi_{i}^{N} d_{i}} \otimes_{j}^{N} I_{d_{j}}+\sum_{M=1}^{N} \sum_{\left\{\mu_{1} \mu_{2} \cdots \mu_{M}\right\}} \sum_{\alpha_{1} \alpha_{2} \cdots \alpha_{M}} \mathcal{T}_{\alpha_{1} \alpha_{2} \cdots \alpha_{M}}^{\left\{\mu_{1} \mu_{2} \cdots \mu_{M}\right\}} \otimes_{n=1}^{M} U_{\mu_{n}} \lambda_{\alpha_{1}}^{\left\{\mu_{1}\right\}} \lambda_{\alpha_{2}}^{\left\{\mu_{2}\right\}} \cdots \lambda_{\alpha_{M}}^{\left\{\mu_{M}\right\}} \otimes_{n=1}^{M} U_{\mu_{n}}^{\dagger} \\
& =\frac{1}{\Pi_{i}^{N} d_{i}} \otimes_{j}^{N} I_{d_{j}}+\sum_{M=1}^{N} \sum_{\left\{\mu_{1} \mu_{2} \cdots \mu_{M}\right\}} \sum_{\alpha_{1} \cdots \alpha_{M} \beta_{1} \cdots \beta_{M}} \mathcal{T}_{\alpha_{1} \alpha_{2} \cdots \alpha_{M}}^{\left\{\mu_{1} \mu_{2} \cdots \mu_{M}\right\}} O_{\alpha_{1} \beta_{1}}^{\mu_{1}} O_{\alpha_{2} \beta_{2}}^{\mu_{2}} \cdots O_{\alpha_{M} \beta_{M}}^{\mu_{M}} \lambda_{\beta_{1}}^{\left\{\mu_{1}\right\}} \lambda_{\beta_{2}}^{\left\{\mu_{2}\right\}} \cdots \lambda_{\beta_{M}}^{\left\{\mu_{M}\right\}}
\end{aligned}
$$

Since $\rho^{\prime}$ can also be written in the form of (11), we obtain that

$$
\mathcal{T}_{\beta_{1} \cdots \beta_{M}}^{\prime\left\{\mu_{1} \cdots \mu_{M}\right\}}=\sum_{\alpha_{1} \cdots \alpha_{M}} O_{\alpha_{1} \beta_{1}}^{\mu_{1}} \cdots O_{\alpha_{M} \beta_{M}}^{\mu_{M}} \mathcal{T}_{\alpha_{1} \cdots \alpha_{M}}^{\left\{\mu_{1} \cdots \mu_{M}\right\}} .
$$

There must exist orthogonal operators $O^{1}, O^{2}, \cdots, O^{N}$ such that for any $1<M \leq N$,

$$
\mathcal{T}^{\left\{\mu_{1} \mu_{2} \cdots \mu_{M}\right\}}=\otimes_{n=1}^{M} O^{\mu_{n}} \mathcal{T}^{\left\{\mu_{1} \mu_{2} \cdots \mu_{M}\right\}} .
$$


By virtue of Proposition 2 in [22] and the analysis therein, one has that the core tensor of the high order tensors $\mathcal{T}^{\left\{\mu_{1} \mu_{2} \cdots \mu_{M}\right\}}$ and $\mathcal{T}^{\left\{\mu_{1} \mu_{2} \cdots \mu_{M}\right\}}$ must be the same up to the local symmetry $\otimes_{n=1}^{M} P_{\left\{\mu_{1} \mu_{2} \cdots \mu_{M}\right\}}^{\mu_{n}}$.

Let

$$
\mathcal{T}^{\left\{\mu_{1} \mu_{2} \cdots \mu_{M}\right\}}=\otimes_{n=1}^{M} U_{\left\{\mu_{1} \mu_{2} \cdots \mu_{M}\right\}}^{\mu_{n}} \Sigma^{\left\{\mu_{1} \mu_{2} \cdots \mu_{M}\right\}}
$$

be the high order singular value decomposition of $\mathcal{T}^{\left\{\mu_{1} \mu_{2} \cdots \mu_{M}\right\}}$. We are readily to present out main result.

Theorem: For N-qubit quantum systems, $\Sigma^{\left\{\mu_{1} \mu_{2} \cdots \mu_{M}\right\}}$ with $\left\{\mu_{1} \mu_{2} \cdots \mu_{M}\right\} \subset\{1,2, \cdots, N\}$ give a local unitary classification up to the local symmetry $\otimes_{n=1}^{M} P_{\left\{\mu_{1} \mu_{2} \cdots \mu_{M}\right\}}^{\mu_{n}}$. For $2 \leq M \leq N, P_{\left\{\mu_{1} \mu_{2} \cdots \mu_{M}\right\}}^{\mu_{n}}$ are formed by a set of orthogonal matrices $\left\{V_{\left\{\mu_{1} \mu_{2} \cdots \mu_{M}\right\}}^{\mu_{n}}\right\}$ and $U_{\left\{\mu_{1} \mu_{2} \cdots \mu_{M}\right\}}^{\mu_{n}}$ such that

$$
P_{\left\{\mu_{1} \mu_{2} \cdots \mu_{M}\right\}}^{\mu_{n}}=\left(V_{\left\{\mu_{1} \mu_{2} \cdots \mu_{M}\right\}}^{\mu_{n}}\right)^{\dagger} P_{\left\{\mu_{1}\right\}}^{\mu_{1}} U_{\left\{\mu_{1} \mu_{2} \cdots \mu_{M}\right\}}^{\mu_{n}} .
$$

Proof: From the double cover relation between $S U(2)$ and $S O(3)$, we have that $\rho$ and $\rho^{\prime}$ are local unitary equivalent if and only if there exist orthogonal matrices $O^{1}, O^{2}, \cdots, O^{N}$ such that (6) hold for any $\left\{\mu_{1} \mu_{2} \cdots \mu_{M}\right\} \subset\{1,2, \cdots, N\}$.

Denoting

$$
\mathcal{T}^{\prime\left\{\mu_{1} \mu_{2} \cdots \mu_{M}\right\}}=\otimes_{n=1}^{M} V_{\left\{\mu_{1} \mu_{2} \cdots \mu_{M}\right\}}^{\mu_{n}} \Lambda^{\left\{\mu_{1} \mu_{2} \cdots \mu_{M}\right\}}
$$

the high order singular decompositions of the tensors $\mathcal{T}^{\left\{\mu_{1} \mu_{2} \cdots \mu_{M}\right\}}$ and noticing (6) and (7) we have that

$$
\Lambda^{\left\{\mu_{1} \cdots \mu_{M}\right\}}=\otimes_{n=1}^{M}\left(V_{\left\{\mu_{1} \cdots \mu_{M}\right\}}^{\mu_{n}}\right)^{\dagger} O^{\mu_{n}} U_{\left\{\mu_{1} \cdots \mu_{M}\right\}}^{\mu_{n}} \Sigma^{\left\{\mu_{1} \cdots \mu_{M}\right\}} .
$$

Define

$$
P_{\left\{\mu_{1} \mu_{2} \cdots \mu_{M}\right\}}^{\mu_{n}}=\left(V_{\left\{\mu_{1} \mu_{2} \cdots \mu_{M}\right\}}^{\mu_{n}}\right)^{\dagger} O^{\mu_{n}} U_{\left\{\mu_{1} \mu_{2} \cdots \mu_{M}\right\}}^{\mu_{n}}
$$

and $O^{\mu_{n}}=P_{\left\{\mu_{1}\right\}}^{\mu_{n}}$. According to the proposition 2 in [22] and the proof therein, we have that $P_{\left\{\mu_{1} \mu_{2} \cdots \mu_{M}\right\}}^{\mu_{n}}$ must be some local symmetric operators.

On the other hand, if $\Lambda^{\left\{\mu_{1} \mu_{2} \cdots \mu_{M}\right\}}$ and $\Sigma^{\left\{\mu_{1} \mu_{2} \cdots \mu_{M}\right\}}$ are related by some $P_{\left\{\mu_{1} \mu_{2} \cdots \mu_{M}\right\}}^{\mu_{1}}$ with the conditions listed in the theorem, by selecting the set of orthogonal matrices $V_{\left\{\mu_{1} \mu_{2} \cdots \mu_{M}\right\}}^{\mu_{n}}$ to be the ones in the high order singular value decompositions of $\mathcal{T}^{\left\{\mu_{1} \mu_{2} \cdots \mu_{M}\right\}}$, one finds that

$$
O^{\mu_{n}}=V_{\left\{\mu_{1} \mu_{2} \cdots \mu_{M}\right\}}^{\mu_{n}} P_{\left\{\mu_{1} \mu_{2} \cdots \mu_{M}\right\}}^{\mu_{n}}\left(U_{\left\{\mu_{1} \mu_{2} \cdots \mu_{M}\right\}}^{\mu_{n}}\right)^{\dagger}=P_{\left\{\mu_{1}\right\}}^{\mu_{n}}
$$

depends only on $\mu_{n}$. Therefore we have (6) holds for any $\left\{\mu_{1} \mu_{2} \cdots \mu_{M}\right\} \subset\{1,2, \cdots, N\}$, which ends the proof.
The theorem provides a practical protocol to verify whether two $N$-qubit mixed states $\rho$ and $\rho^{\prime}$ are local unitary equivalent or not:

(1) First write $\rho$ and $\rho^{\prime}$ in the generalized Bloch representations (11).

(2) Compute the high order singular decompositions of the tensors (7) and (9). Find all the singular values for $\left\{\mu_{1} \mu_{2} \cdots \mu_{M}\right\} \subset\{1,2, \cdots, N\}$. Since the tensors we refer here are all with sub dimensional 3 , the computation of the high order singular value decomposition can be very simple. If the corresponding singular values are not the same, $\rho$ and $\rho^{\prime}$ are not local unitary equivalent.

(3) Otherwise, for any $M \in\{1,2, \cdots, N\}$, check if there exist some proper $P_{\left\{\mu_{1} \mu_{2} \cdots \mu_{M}\right\}}^{\mu_{n}}$ such that

$$
\Sigma^{\left\{\mu_{1} \mu_{2} \cdots \mu_{M}\right\}}=\otimes_{n=1}^{M} P_{\left\{\mu_{1} \mu_{2} \cdots \mu_{M}\right\}}^{\mu_{n}} \Lambda^{\left\{\mu_{1} \mu_{2} \cdots \mu_{M}\right\}}
$$

and (6) hold, where

$$
O^{\mu_{n}}=V_{\left\{\mu_{1} \mu_{2} \cdots \mu_{M}\right\}}^{\mu_{n}} P_{\left\{\mu_{1} \mu_{2} \cdots \mu_{M}\right\}}^{\mu_{n}}\left(U_{\left\{\mu_{1} \mu_{2} \cdots \mu_{M}\right\}}^{\mu_{n}}\right)^{\dagger}
$$

are orthogonal operators depending on the $\mu_{n}$ th subsystem only. If no such $P_{\left\{\mu_{1} \mu_{2} \cdots \mu_{M}\right\}}^{\mu_{n}}$ can be found, $\rho$ and $\rho^{\prime}$ are not local unitary equivalent.

Searching the local symmetries for multi-qubit systems: From the above procedures, we see that, since the expression of the core tensor is only unique up to some local symmetries, the problem becomes very hard to implement the LU classification. Such problems exist too in 22] for the case of multi-qudit pure states. Fortunately, by using the iterative property of the conditions satisfied by the core tensors and taking into account that $\mathcal{T}^{\left\{\mu_{1} \mu_{2} \cdots \mu_{M}\right\}}$ are real, we are able to propose a rather simple way to do the step (3) above operationally. In the following we give a detailed explanation for searching the local symmetries for multi-qubit mixed states.

We start with the first order tensors

$$
\Lambda^{\left\{\mu_{1}\right\}}=\mathcal{T}^{\prime\left\{\mu_{1}\right\}}=P^{\mu_{1}} \Sigma^{\left\{\mu_{1}\right\}}=P^{\mu_{1}} \mathcal{T}^{\left\{\mu_{1}\right\}} .
$$

Any local symmetry $P^{\mu_{1}}$ transform $\Sigma^{\left\{\mu_{1}\right\}}$ to $\Lambda^{\left\{\mu_{1}\right\}}$ can be represented as a rotation. Since the first order tensors $\Lambda^{\left\{\mu_{1}\right\}}$ and $\Sigma^{\left\{\mu_{1}\right\}}$ are 3 dimensional vectors, $P^{\mu_{1}}$ is a rotation of $S O(3)$ group with three independent parameters. From $\Lambda^{\left\{\mu_{1}\right\}}=P^{\mu_{1}} \Sigma^{\left\{\mu_{1}\right\}}$, we can reduce the free parameters in $P^{\mu_{1}}$ from three to one. Correspondingly, $O^{\mu_{1}}=P^{\mu_{1}}$.

From the condition of the second order tensor, one has $P_{\left\{\mu_{1} \mu_{2}\right\}}^{\mu_{1}}=\left(V_{\left\{\mu_{1} \mu_{2}\right\}}^{\mu_{1}}\right)^{\dagger} O^{\mu_{1}} U_{\left\{\mu_{1} \mu_{2}\right\}}^{\mu_{1}}=\left(V_{\left\{\mu_{1} \mu_{2}\right\}}^{\mu_{1}}\right)^{\dagger} P^{\mu_{1}} U_{\left\{\mu_{1} \mu_{2}\right\}}^{\mu_{1}}$. The free one parameter in $P^{\mu_{1}}$ is further determined by the equations

$$
\Sigma^{\left\{\mu_{1} \mu_{2}\right\}}=P_{\left\{\mu_{1} \mu_{2}\right\}}^{\mu_{1}} \otimes P_{\left\{\mu_{1} \mu_{2}\right\}}^{\mu_{2}} \Lambda^{\left\{\mu_{1} \mu_{2}\right\}} .
$$


Then one checks the third order tensor's condition, the fourth one, ... , and all the conditions iteratively. If at least a set of $\left\{P^{\mu_{n}}, n=1,2, \cdots, N\right\}$ satisfies all the conditions in the above procedures, then we conclude that $\rho$ and $\rho^{\prime}$ are LU equivalent. Or we get a negative answer. The corresponding $U_{1}, U_{2}, \cdots, U_{N}$ can be immediately computed by using the double cover relation between $S U(2)$ and $S O(3)$ (parameterized as that in [25]).

Generally, for any given multi-qubit quantum state $\rho$, we are able to find the equivalent class of $\rho$ under LU. We first compute all the tensors $\mathcal{T}^{\left\{\mu_{1} \mu_{2} \cdots \mu_{M}\right\}}$ and the corresponding core tensors $\Sigma^{\left\{\mu_{1} \mu_{2} \cdots \mu_{M}\right\}}$, where $1 \leq M \leq$ $N$. Then we parameterize the $3 \times 3$ orthogonal matrix $\left\{P_{\left\{\mu_{1}\right\}}^{\mu_{n}}, n=1,2, \cdots, N\right\}$ and define (8) for $2 \leq M \leq N$ and for any $V_{\left\{\mu_{1} \mu_{2} \cdots \mu_{M}\right\}}^{\mu_{n}}$. According to the theorem, all the $\left\{P_{\left\{\mu_{1} \mu_{2} \cdots \mu_{M}\right\}}^{\mu_{n}}, n=1,2, \cdots, N\right\}$ and the core tensors $\Sigma\left\{\mu_{1} \mu_{2} \cdots \mu_{M}\right\}$ form the class of quantum states that are LU equivalent to $\rho$.

Example: Consider two mixed three-qubit quantum states: $\rho=\frac{2}{17}\left(2\left|\psi_{+}\right\rangle\left\langle\psi_{+}|+| 001\right\rangle\langle 001|+| 010\rangle\langle 010|+\right.$ $\left.2|011\rangle\left\langle 011\left|+\frac{1}{2}\right| 100\right\rangle\langle 100|+| 101\rangle\langle 101|+| 110\rangle\langle 110|\right)$ with $\left|\psi_{+}\right\rangle=\frac{1}{\sqrt{2}}(|000\rangle+|111\rangle)$ and

$$
\sigma=\frac{2}{17}\left(\begin{array}{cccccccc}
1 & 0 & 0 & 0 & 0 & \frac{1}{2} & 0 & \frac{1}{2} \\
0 & \frac{3}{2} & 0 & \frac{1}{2} & 0 & 0 & 0 & 0 \\
0 & 0 & 1 & 0 & 0 & -\frac{1}{2} & 0 & -\frac{1}{2} \\
0 & \frac{1}{2} & 0 & \frac{3}{2} & 0 & 0 & 0 & 0 \\
0 & 0 & 0 & 0 & \frac{3}{4} & 0 & \frac{1}{4} & 0 \\
\frac{1}{2} & 0 & -\frac{1}{2} & 0 & 0 & 1 & 0 & 0 \\
0 & 0 & 0 & 0 & \frac{1}{4} & 0 & \frac{3}{4} & 0 \\
\frac{1}{2} & 0 & -\frac{1}{2} & 0 & 0 & 0 & 0 & 1
\end{array}\right) .
$$

The eigenvalues of $\rho$ and $\sigma$ are both $\left\{\frac{2}{17}, \frac{2}{17}, \frac{2}{17}, \frac{2}{17}, \frac{4}{17}, \frac{4}{17}, \frac{1}{17}, 0\right\}$, which means that the two states we considering are degenerate. The corresponding correlation tensors are listed below:

$$
\begin{aligned}
& \mathcal{T}^{\{1\}}=\left(0,0,-\frac{3}{17}\right) \\
& \mathcal{T}^{\{2\}}=\mathcal{T}^{\{3\}}=\left(0,0, \frac{3}{17}\right) \\
& \mathcal{T}^{\{12\}}=\mathcal{T}^{\{13\}}=\left(0,0,0,0,0,0,0,0,-\frac{1}{17}\right),
\end{aligned}
$$

$$
\mathcal{T}^{\{23\}}=\left(0,0,0,0,0,0,0,0, \frac{1}{17}\right)
$$

$$
\begin{aligned}
\mathcal{T}^{\{123\}}= & \left(\frac{4}{17}, 0,0,0,-\frac{4}{17}, 0,0,0,0,0,-\frac{4}{17}, 0,-\frac{4}{17},\right. \\
& \left.0,0,0,0,0,0,0,0,0,0,0,0,0,-\frac{3}{17}\right)
\end{aligned}
$$

$$
\begin{gathered}
\mathcal{T}^{\prime\{1\}}=\left(0,0,-\frac{3}{17}\right), \mathcal{T}^{\prime\{2\}}=\left(\frac{3}{17}, 0,0\right), \\
\mathcal{T}^{\prime\{3\}}=\left(0,0, \frac{3}{17}\right)
\end{gathered}
$$

$$
\mathcal{T}^{\prime\{12\}}=\left(0,0,0,0,0,0,-\frac{1}{17}, 0,0\right)
$$

$$
\mathcal{T}^{\prime\{13\}}=\left(0,0,0,0,0,0,0,0,-\frac{1}{17}\right)
$$

$$
\begin{aligned}
\mathcal{T}^{\prime\{23\}}=\left(0,0, \frac{1}{17}, 0,0,0,0,0,0\right) \\
\mathcal{T}^{\prime\{123\}=}\left(0,0,0,0,-\frac{4}{17}, 0,-\frac{4}{17}, 0,0,0,0,0,-\frac{4}{17} 0\right. \\
\left.\quad 0,0, \frac{4}{17}, 0,0,0,-\frac{3}{17}, 0,0,0,0,0,0\right) .
\end{aligned}
$$

One can check that all the correlation tensors are just in their core tensor form. And all the corresponding singular values are the same. So we come to the step (3) to detect the LU equivalence of $\rho$ and $\sigma$.

To derive $P^{1}, P^{2}$ and $P^{3}$, we parameterize any $2 \times 2$ unitary matrix by sketching the representation in [25] as

$$
U=\left(\begin{array}{cc}
\cos \theta e^{i \phi} & \sin \theta e^{i \chi} \\
-\sin \theta e^{-i \chi} & \cos \theta e^{-i \phi}
\end{array}\right)
$$

The corresponding orthogonal matrix derived in (5) can be written as

$$
P=\left(\begin{array}{ccc}
\cos ^{2}(\theta) \cos (2 \phi)-\sin ^{2}(\theta) \cos (2 \chi) & \cos ^{2}(\theta) \sin (2 \phi)+\sin ^{2}(\theta) \sin (2 \chi) & -\sin (2 \theta) \cos (\phi+\chi) \\
\sin ^{2}(\theta) \sin (2 \chi)-\cos ^{2}(\theta) \sin (2 \phi) & \cos ^{2}(\theta) \cos (2 \phi)+\sin ^{2}(\theta) \cos (2 \chi) & \sin (2 \theta) \sin (\phi+\chi) \\
\sin (2 \theta) \cos (\chi-\phi) & -\sin (2 \theta) \sin (\chi-\phi) & \cos (2 \theta)
\end{array}\right)
$$

By solving that $\mathcal{T}^{\prime\{1\}}=P^{1} \mathcal{T}^{\{1\}}, \mathcal{T}^{\prime\{2\}}=P^{2} \mathcal{T}^{\{2\}}$, and $\mathcal{T}^{\prime\{3\}}=P^{3} \mathcal{T}^{\{3\}}$, one obtains the corresponding parame- 
ters to be $\left\{\theta_{1}=0\right\},\left\{\theta_{2}=\frac{\pi}{4}, \phi_{2}=\pi-\chi_{2}\right\}$ and $\left\{\theta_{3}=0\right\}$. Then we simplify $P^{1}, P^{2}$ and $P^{3}$ to be

$$
\begin{aligned}
P^{1} & =\left(\begin{array}{ccc}
\cos \left(2 \phi_{1}\right) & \sin \left(2 \phi_{1}\right) & 0 \\
-\sin \left(2 \phi_{1}\right) & \cos \left(2 \phi_{1}\right) & 0 \\
0 & 0 & 1
\end{array}\right), \\
P^{2} & =\left(\begin{array}{ccc}
0 & 0 & 1 \\
\sin (2 \chi) & \cos (2 \chi) & 0 \\
-\cos (2 \chi) & \sin (2 \chi) & 0
\end{array}\right), \\
P^{3} & =\left(\begin{array}{ccc}
\cos \left(2 \phi_{3}\right) & \sin \left(2 \phi_{3}\right) & 0 \\
-\sin \left(2 \phi_{3}\right) & \cos \left(2 \phi_{3}\right) & 0 \\
0 & 0 & 1
\end{array}\right) .
\end{aligned}
$$

By the rest conditions that the second and third order core tensors should satisfy, i.e.

$$
\begin{aligned}
& \mathcal{T}^{\prime\{12\}}=P^{1} \otimes P^{2} \mathcal{T}^{\{12\}} \\
& \mathcal{T}^{\prime\{13\}}=P^{1} \otimes P^{3} \mathcal{T}^{\{13\}} ; \\
& \mathcal{T}^{\prime\{23\}}=P^{2} \otimes P^{3} \mathcal{T}^{\{23\}}
\end{aligned}
$$

and

$$
\mathcal{T}^{\prime\{123\}}=P^{1} \otimes P^{2} \otimes P^{3} \mathcal{T}^{\{123\}},
$$

one can find the final solutions for $P^{1}, P^{2}$ and $P^{3}$ with parameters satisfying $\phi_{1}-\chi_{2}+\phi_{3}=0$.

Finally we obtain that the survival local symmetries $P^{1}, P^{2}$ and $P^{3}$ must be with the conditions $\left\{\theta_{1}=0\right\},\left\{\theta_{2}=\frac{\pi}{4}, \phi_{2}=\pi-\chi_{2}\right\},\left\{\theta_{3}=0\right\}$ and $\phi_{1}-\chi_{2}+\phi_{3}=0$. For example, one can select $\theta_{1}=\phi_{1}=0, \theta_{2}=\frac{\pi}{4}, \phi_{2}=\pi, \chi_{2}=0$ and $\theta_{3}=\phi_{3}=0$. Then we have that $P^{1}=P^{3}=I$ and $P^{2}$ be an anti-diagonal matrix with entries $\{1,1,-1\}$. The corresponding local unitary operators that transform $\rho$ to $\sigma$ are then computed to be $U_{1}=U_{3}=I$ and $U_{2}=\left\{\left\{\frac{\sqrt{2}}{2} e^{i \pi}, \frac{\sqrt{2}}{2}\right\},\left\{-\frac{\sqrt{2}}{2}, \frac{\sqrt{2}}{2} e^{-i \pi}\right\}\right\}$.

Conclusions and Remarks: It is a basic and fundamental question to classify quantum states under local unitary operations. The problem has been figured out in 21, 22] for pure multipartite quantum states. However, it is much more difficult to classify mixed quantum states under LU transformations than that for pure states. Operational methods have been presented only for non-degenerated bipartite states. Although the authors in [29] have shown that the problem of mixed states can be reduced to the one of pure states in terms of the purification of mixed states mathematically, the protocol is rather not operational. In this letter we have provided an operational way to verify and classify quantum states by using the generalized Bloch representation in terms of the generators of $S U(d)$. Since the tensors in the representation can be all determined by some local quantum mechanical observables, the method is experimentally feasible. Our criterion is both sufficient and necessary for multi-qubit quantum systems, thus gives rise to a complete classification of multi-qubit quantum states under LU transformations.

Acknowledgments This work is supported by the NSFC 11105226, 11275131; the CSC; the Fundamental Research Funds for the Central Universities No.12CX04079A, No.24720122013; Research Award Fund for outstanding young scientists of Shandong Province No.BS2012DX045.

[1] M.A. Nielsen and I.L. Chuang, Quantum Computation and Quantum Information. Cambridge: Cambridge University Press, (2000).

[2] D. Gottesman, Ph.D. thesis, Caltech arXiv:quant-ph/ 9705052.

[3] R. Raussendorf and H. J. Briegel, Phys. Rev. Lett. 86, 5188 (2001).

[4] L. Amico et al., Rev. Mod. Phys. 80, 517 (2008); F. Verstraete et al., Adv. Phys. 57, 143 (2008), and references therein.

[5] R. Horodecki et al., Rev. Mod. Phys. 81, 865 (2009).

[6] C. H. Bennett, D. P. DiVincenzo, J. A. Smolin, and W. K. Wootters, Phys. Rev. A 54, 3824 (1996); C. H. Bennett, H. J. Bernstein, S. Popescu, and B. Schumacher, ibid. 53, 2046 (1996); V. Vedral, M. B. Plenio, M. A. Rippin, and P. L. Knight, Phys. Rev. Lett. 78, 2275 (1997).

[7] W. K. Wootters, Phys. Rev. Lett. 80, 2245 (1998).

[8] R. Horodecki, P. Horodecki, and M. Horodecki, Phys. Lett. A 200, 340 (1995).

[9] R. F. Werner and M. M. Wolf, Phys. Rev. A 64, 032112 (2001).

[10] M. Zukowski and C. Brukner, Phys. Rev. Lett. 88, 210401 (2002).

[11] M. Li and S.M. Fei, Phys. Rev. A 86, 052119 (2012).

[12] M. Horodecki, P. Horodecki, and R. Horodecki, Phys. Rev. A, 60, 1888(1999).

[13] S. Albeverio, S. M. Fei, and W. L. Yang, Phys. Rev. A, 66, 012301(2002).

[14] Y. Makhlin, Quant. Info. Proc. 1, 243 (2002).

[15] N. Linden, S. Popescu and A. Sudbery, Phys. Rev. Lett. 83, 243 (1999).

[16] N. Linden and S. Popescu, Fortsch. Phys. 46, 567 (1998).

[17] S. Albeverio, S.M. Fei, P.Parashar,W.L.Yang, Phys. Rev. A 68, 010303 (2003).

[18] S. Albeverio, S.M. Fei, and D.Goswami, Phys. Lett. A 340, 37 (2005).

[19] B.Z. Sun, S.M. Fei, X.Q. Li-Jost and Z.X.Wang, J. Phys. A 39, L43-L47(2006).

[20] S. Albeverio, L. Cattaneo, S.M. Fei and X.H. Wang, Int. J. Quant. Inform. 3, 603 (2005).

[21] B. Kraus, Phys. Rev. Lett. 104, 020504(2010); Phys. Rev. A 82, 032121 (2010).

[22] B. Liu, J.L. Li, X. Li, C.F. Qiao, Phys. Rev. Lett.108, 050501 (2012).

[23] C. Zhou, T.G. Zhang, S.M. Fei, N. Jing, and X. Li-Jost, Phys. Rev. A 86(R), 010303 (2012).

[24] T.G. Zhang, M.J. Zhao, M. Li, S.M. Fei, and X. Li-Jost, Phys. Rev. A, 88, 042304 (2013)

[25] J. Schlienz and G. Mahler, Phys. Rev. A 52, 4396 (1995). 
[26] A. Saif M. Hassan and Pramod S. Joag, Quant. Info. Comput. 8, 0773(2008).

[27] L. D. Lathauwer, B.D. Moor, and J. Vandewalle, SIAM J. Matrix Anal. Appl.21, 1253(2000);

Tamara G. Kolda and Brett W. Bader, SIAM Rev.51, 455(2009).
[28] W. Dur, G. Vidal, and J.I. Cirac, Phys. Rev. A 62, 062314 (2000).

[29] J.L. Li and C.F. Qiao, J. Phys. A: Math. Theor. 46, 075301 (2013). 